\title{
EFECTOS DE LA TENSIÓN CALÓRICA EN LA ÉPOCA SECA SOBRE ALGUNOS ÍNDICES FISIOLÓGICOS Y EL ESTADO GENERAL EN LACTACIÓN Y CRECIMIENTO EN GANADO LECHERO CRUZADO*
}

\author{
Edil E. Arauz**
}

\begin{abstract}
RESUMEN
El presente estudio se hizo con el propósito cuantificar las principales variables climáticas y su efecto sobre algunos índices fisiológicos, durante la lactancia y crecimiento del ganado lechero híbrido en la época seca de las zonas costeras de Panamá. La prueba se realizó en las instalaciones del Programa Lechero de Doble Propósito de la Universidad de Panamá, en Chiriquí, Panamá. Las variables fisiológicas fueron analizadas empleando el diseño de parcela subdividida en tiempo y contraste de regresión polinomiales. Se utilizaron total de 10 vacas híbridas en lactancia con un peso promedio de $446 \mathrm{~kg}$ y una producción láctea de $6,80 \mathrm{~kg} / \mathrm{vaca} /$ día. así como 10 terneros con peso de $118 \mathrm{~kg}$ los animales eran cruzas de Holstein-Pardo Suizo con Cebú. La caracterización microclimática basada en la radiación solar, temperatura ambiental e índice temperatura-humedad indicó que el período diurno de la época seca es representativo de la tensión calórica efectiva de tipo climático, en especial entre 10:00 am y 4:pm. Las vacas y los terneros fueron afectados por la tensión calórica, por lo cual la temperatura rectal y el índice respiratorio sufrieron incrementos, cuadrático y cúbico, respectivamente, sobre el patrón fisiológico.
\end{abstract}

\begin{abstract}
The test was conducted, at the facilities of the Dual Purpose Dairy Program of the University of Panama in Chiriqui, Panama, to quantify the main climatic variables during the dry season and their effect on so me physiological indexes of lactating and growing hybrid dairy cattle. The physiological variables were analyzed using the split-plot experimental design in time and contrast of polynomial regression. Ten lactating hybrid cows, with and average weight of $446 \mathrm{~kg}$ and a milk production of $6.8 \mathrm{~kg} / \mathrm{cow} /$ day, and ten calves weighing $118 \mathrm{~kg}$ each were used. The animals were Holstein/Brown Swiss X Zebu crossbreeds. The micro-climatic characterization, based on solar radiation, ambient temperature and the temperature-humidity index, indicated that the day time period during the dry season is the representative type of effective climatic heat stress, lactating cows and calves were affected by heat stress, and quadratic and cubic increases were observed in rectal temperature and the respiratory index over the normal physiological pattern.
\end{abstract}

\section{INTRODUCCIÓN}

Se reconoce que el ganado lechero es muy exigente en condiciones climáticas debido a su origen, objetivo de producción y caracteres metabólicos y morfo fisiológicos. Por ello la importancia de la Tensión Calórica, lo cual significa un elemento negativo sobre la producción lechera, en particular durante la época seca a nivel de los trópicos y subtrópicos. Considerando los criterios más fundamentales de la Tensión Calórica y sus efectos en la producción de leche y el desempeño del ganado lechero es que se han empleado los llamados cruzamientos de ganado lechero europeo x cebú (e.g. Holstein x Cebú y Pardo Suizo x Cebú) buscando un animal con cierta aptitud lechera, pero con mayor tolerancia a la inclemencia climática del trópico. Sin embargo, a pesar de que se reconocen las implicaciones negativas de la época seca sobre el desempeño de los bovinos de leche en los trópicos y subtrópicos, aún es necesario determinar o cuantificar la contribución del complejo climático sobre el desempeño fisiológico inmediato en el ganado lechero cruzado o acebunado.

El estado de tensión calórica está determinado por altos índices de radiación solar directa, temperatura ambiental, temperatura del suelo (superficie), índice temperatura-humedad (complejo), presión de vapor de agua y humedad relativa (Curtis, 1981; Folk, 1973). Todos estos elementos definen en conjunto el complejo microclimático de tensión calórica, el cual influye directamente sobre la fisiología general (Folk, 1973), producción animal (Hafez, 1973; Collier et al., 1982), reproducción y salud (Curtis, 1981; Anderson, 1984). No obstante, el principal efecto inmediato de la Tensión Calórica ocurre sobre los mecanismos de ajuste y regulación homeostática, lo cual implica cambios en el

\footnotetext{
* Trabajo presentado a la XXXVII Reunión Anual del PCCMCA. Panamá. 1991.

** Profesor Investigador. Departamento de Zootecnia, Facultad de Ciencias Agropecuarias, Universidad de Panamá. Apartado 2B, Ciudad de David, Provincia de Chiriquí. República de Panamá.
} 
funcionamiento de órganos, glándulas y sistemas. Esto se refleja además en cambios metabólicos, supresión y/o reducción de funciones corporales no vitales (e.g. crecimiento, lactación) y aumento significativo de las funciones vinculadas con el mantenimiento funcional del medio interno (Anderson, 1984; Thompson, 1985; Yousef, 1985).

Entre los principales parámetros en alterarse en los bovinos se encuentran la temperatura rectal y el índice respiratorio (Bianca et al 1985; Curtis, 1981; Reece, 1984). Al respecto, Collier et al. (1978), encontró que el ganado lechero en lactación sufre drástica alteración en la magnitud de la temperatura rectal y la frecuencia respiratoria durante el verano. En efecto, el aumento de la frecuencia respiratoria por un prolongado per íodo, provoca una disminución excesiva del $\mathrm{CO}_{2}$ en la sangre debido a la hiperventilación pulmonar, lo cual conduce a un estado de alcalosis espiratoria, dándose un desbalance del estado ácido-base en la sangre (Curtis, 1981; Bullock et al., 1984).

Los cambios que se producen en el índice respiratorio y la temperatura rectal son un reflejo de los ajustes integrados que se dan en el animal bajo influencia de la tensión calórica para aumentar la pérdida de calor en forma activa, lo cual es efectivo biológicamente siempre que la temperatura ambiental y la humedad relativa no interfieran con el proceso de evaporación (Hafez, 1973; Yousef, 1985).

Frente a los efectos inmediatos y a largo plazo sobre los bovinos de leche, se han planteado una serie de alternativas de manejo y alimentación para sobrellevar la producción de leche, sin tener mayores repercuciones en el desempeño productivo (Beede et al., 1986; Collier et al., 1978), a pesar de que genética y climatológicamente continúan existiendo diversas interrogantes según la región, el país, tipo de animal empleado y el nivel explotacional.

El presente estudio fue efectuado con el propósito de cuantificar las principales variables climáticas (radiación solar directa, humedad relativa y temperatura ambiental) y fisiológicas (temperatura rectal, índice respiratorio y cardíaco) para asociar las mismas y definir el impacto inmediato sobre el desempeño físiológico en el ganado lechero cruzado en crecimiento y lactación, a nivel de zonas costeras en el per íodo diurno de la época seca.

\section{MATERIALES Y METODOS}

El presente estudio se realizó en 1989 en las instalaciones del Programa Lechero de Doble Propósito de la Facultad de Ciencias Agropecuarias, Universidad de Panamá en la provincia de Chiriquí. El área está a 25 msnm, latitud $8^{\circ} 23^{\prime} 57^{\prime \prime} \mathrm{N}$ y longitud entre $82^{\circ} 19^{\prime} 47^{\prime \prime}$ y $82^{\circ} 20^{\prime} 47 " \mathrm{~W}$. El experimento se efectuó en el mes de abril de 1989, cuya caracterización climática general fue a fin con los meses de enero, febrero y marzo dentro del concepto de época seca que incluye de diciembre a marzo.

Se utilizaron un total de 10 vacas cruzadas en lactación con peso promedio de $446 \mathrm{~kg}$ y una producción láctea de $6,80 \mathrm{~kg} / \mathrm{vaca} /$ di con 3,28\% de grasa en la leche; así como 10 terneros con peso de $118 \mathrm{~kg}$. Se utilizaron animales con influencia de ganado lechero europeo (Holstein, Pardo Suizo) y Cebú respectivamente, pero sin cruzamientos definidos (e.g. 3/4 H/S x 1/4 Cebú), por lo cual los animales eran identificables con lo que se encuentra a nivel de productor.

El experimento consistió en someter todos los animales a la exposición microclimática durante el día sin suministrar sombra, pero si agua y alimento (heno, melaza, urea, sal mineralizada). Para ello, el per íodo diurno se subdividió en siete subper íodos según la hora diurna de 6 am, 8, 10, 12, 4 Y 6 pm. En cada una de estas horas se midió la temperatura rectal, índice respiratorio y cardíaco; así como las condiciones climáticas (radiación solar, temperatura ambiental, humedad relativa y complejo humedad-temperatura para bovino).

Las variables fisiológicas fueron analizadas empleando el diseño de parcela subdividida en tiempo y contraste de regresión polinominales según lo descrito por Gill (1978).

El modelo principal usado fue:

$$
\begin{aligned}
& \text { Yijk = u+yi }+\mathrm{D}(\mathrm{l}) \mathrm{j}+\mathrm{Bk}+(\text { Ó B }) \mathrm{lk}+\mathrm{E}(\mathrm{ijk}) \\
& \text { donde: }
\end{aligned}
$$

Yijk = Variable fisiológica dependiente (e.g. Indice respiratorio en $\mathrm{N}^{\circ} / \mathrm{min}$.) del animal $\mathrm{j}$ th según el estado fisiológico $\mathrm{i}^{\text {th }}$ en el subperíodo diurno $\mathrm{k}^{\text {th }}$.

$\mathrm{u}=$ Media General 
$\mathrm{i}=$ Efecto del estado fisiológico $\mathrm{i}^{\text {th }} / \mathrm{i}^{\text {th }}=1$ (lactación), 2 (crecimiento)

$\mathrm{D}(\mathrm{i}) \mathrm{j}=$ Efecto de los animales $\mathrm{j}^{\text {th }} \mathrm{o}$ Error $\mathrm{a}$.

$\mathrm{Bk}=$ Efecto de los subperíodos diurnos $\mathrm{K}^{\text {th }}\left(\mathrm{K}^{\text {th }}=6 \mathrm{am}\right.$, $8 \mathrm{am}, 10 \mathrm{am}, 12 \mathrm{md}, 2 \mathrm{pm}, 4 \mathrm{pm}$ y $6 \mathrm{pm})$.

(B)ik= Interacción Estado fisiológico x subperíodo diurno.

$\mathrm{E}(\mathrm{ijk})=$ Residuo experimental o Error b.

\section{RESULTADOS Y DISCUSIÓN}

\section{Complejo microclimático Tensión Calórica}

La determinación de la magnitud de los principales elementos climáticos durante el período diurno de la época seca indicó de manera general que a partir de las 10:00 am, hasta las 4:00 pm se presentó la condición de tensión calórica efectiva, de acuerdo con la radiación solar directa, temperatura ambiental (bulbo seco) y complejo humedad temperatura para bovinos (Cuadro 1). El período diurno crítico (10 am-4 pm) indicó una radiación solar directa de 627 a $452,1 \mathrm{Kcal} / \mathrm{m}^{2} \mathrm{~h}$; temperatura ambiental 31,9 a $32,7^{\circ} \mathrm{C}$ y un complejo humedad-temperatura de 27,15 a $28,14^{\circ} \mathrm{C}$. En efecto Curtis (1981) y Yousef (1985), indican que la alta radiación solar y un índice humedad-temperatura superior a 262C definen gran parte de la tensión calórica que produce efectos agudos y crónicos en los bovinos. De acuerdo con el comportamiento de los parámetros climáticos se observaron tres fases de la tensión calórica, es decir, la de incremento, estabilidad y reducción respectivamente (Cuadro 2). Esto indica que el período diurno presentó gran variación en los principales elementos climáticos, pero que el estado de tensión calórica se presentó en forma definida, como es de esperar en la época seca a nivel de los trópicos y subtrópicos.

Cuadro 1. Principales características climatológicas del periodo diurno en la época seca durante la fase experimental*

\begin{tabular}{|c|c|c|c|c|c|c|c|}
\hline \multirow[b]{2}{*}{ Caracteristicas Climáticas } & \multicolumn{4}{|c|}{$S$ U B P E R I O D O S } & \multicolumn{2}{|c|}{ D I U R N O S } & \multirow[b]{2}{*}{$\begin{array}{c}06: 00 \\
\text { P.M. }\end{array}$} \\
\hline & $\begin{array}{c}06: 00 \\
\text { A.M. }\end{array}$ & $\begin{array}{c}08: 00 \\
\text { A.M. }\end{array}$ & $\begin{array}{c}10: 00 \\
\text { A.M. }\end{array}$ & $\begin{array}{c}12: 00 \\
\text { M.D. }\end{array}$ & $\begin{array}{c}02: 00 \\
\text { P.M. }\end{array}$ & $\begin{array}{c}04: 00 \\
\text { P.M. }\end{array}$ & \\
\hline Temperatura Ambiental (bulbo seco) ${ }^{\circ} \mathrm{C}$ & 21,8 & 25,5 & 31,9 & 33,1 & 32,8 & 32,7 & 30,1 \\
\hline Temperatura Ambiental (bulbo $\mathrm{h}$-medo) ${ }^{\circ} \mathrm{C}$ & 21,2 & 22,4 & 24,1 & 24,5 & 26,0 & 25,8 & 23,6 \\
\hline Radiación Solar Directa $\mathrm{kcal} / \mathrm{m} 2, \mathrm{hr}$ & 0 & 212,2 & 627,0 & 805,2 & 77,07 & 452,1 & 66,0 \\
\hline Precipitación pluvial mm & 0 & 0 & 0 & 0 & 0 & 0 & 0 \\
\hline Humedad relativa $\%$ & 98,5 & 89,8 & 55,3 & 50,3 & 66,8 & 66,8 & 75,5 \\
\hline Velocidad del viento $\mathrm{m} / \mathrm{min}$ & 23,8 & 27,8 & 60,1 & 121,0 & 189,0 & 206,0 & 182,1 \\
\hline 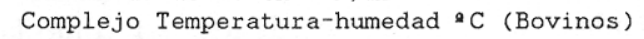 & 22,0 & 24,0 & 27,2 & 27,8 & 28,28 & 28,2 & 26,3 \\
\hline Entalpia Aproximada $\mathrm{kcal} / \mathrm{kg}$ de aire seco & 15,9 & 17,8 & 21,3 & 21,3 & 21,85 & 20,6 & 20,2 \\
\hline
\end{tabular}

* Información obtenida en la Estación Meteorológica de la Facultad de Ciencias Agropecuarias, Universidad de Panamá.

Cuadro 2. Clasificación de la tensión calórica diurna según la magnitud de los elementos climáticos de mayor importancia y los cambios en los patrones fisiológicos en ganado lechero cruzado durante la época seca.

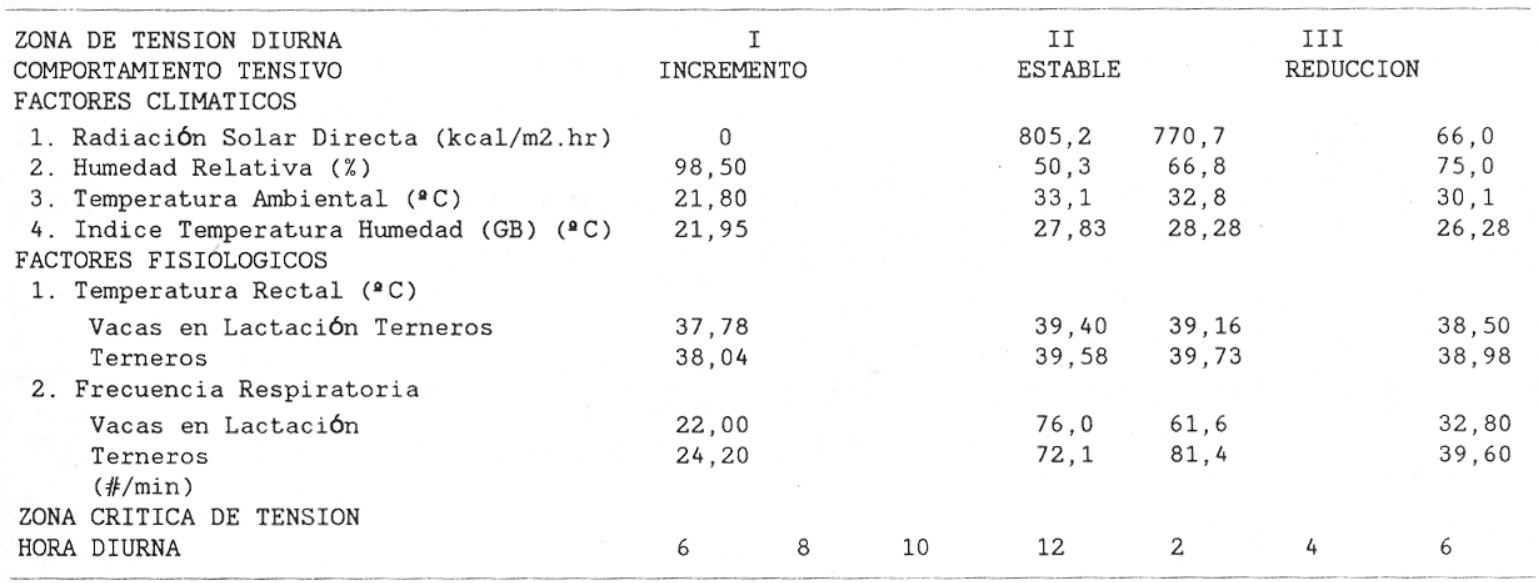


Cuadro 3. Medias de los patrones fisiológicos evaluados en el ganado lechero cruzado en lactación crecimicnto sometidos a tensión calórica durante el período diurno de la época seca.

\begin{tabular}{|c|c|c|c|c|c|c|c|}
\hline \multirow{3}{*}{$\begin{array}{l}\text { Período } \\
\text { Diurno }\end{array}$} & \multirow{3}{*}{$\begin{array}{c}\text { Horas } \\
\text { Diurnas }\end{array}$} & \multicolumn{2}{|c|}{$\begin{array}{c}\text { Temperatura Rectal } \\
\left({ }^{\circ} \mathrm{C}\right)\end{array}$} & \multicolumn{2}{|c|}{$\begin{array}{l}\text { Frecuencia Respiratoria } \\
\text { (No/minuto) }\end{array}$} & \multicolumn{2}{|c|}{$\begin{array}{l}\text { Frecuencia Cardiaca } \\
\text { (No/minuto) }\end{array}$} \\
\hline & & Vacas en & Terneros & Vacas en & Terneros & Vacas en & Terneros \\
\hline & & Lactación & & Lactación & & Lactacion & \\
\hline $1^{*}$ & $06: 00 \mathrm{AM}$ & $37,78 a$ & $38,04 a$ & $22,0 \mathrm{a}$ & $24,2 \mathrm{a}$ & $53,4 \mathrm{a}$ & $52,3 a$ \\
\hline 2 & $08: 00 \mathrm{AM}$ & $38,60 \mathrm{~b}$ & $38,41 \mathrm{a}$ & $41,6 b$ & $32,0 \mathrm{a}$ & $58,4 a$ & $61,2 \mathrm{a}$ \\
\hline 3 & $10: 00 \mathrm{AM}$ & $39,06 \mathrm{~b}$ & $39,48 b$ & $60,8 b$ & $68,3 b$ & $62,0 \mathrm{a}$ & $63,3 \mathrm{a}$ \\
\hline 4 & $12: 00 \mathrm{MD}$ & $39,40 \mathrm{~b}$ & $39,58 b$ & $76,0 \mathrm{~b}$ & $72,1 \mathrm{~b}$ & $65,0 \mathrm{~b}$ & $62,7 a$ \\
\hline 5 & 02:00 PM & $39,16 b$ & $39,73 b$ & $61,6 \mathrm{~b}$ & $81,4 \mathrm{~b}$ & $61,2 \mathrm{a}$ & $61,3 \mathrm{a}$ \\
\hline 6 & $04: 00 \mathrm{PM}$ & $38,92 b$ & $39,62 b$ & $36,4 \mathrm{c}$ & $74,1 \mathrm{~b}$ & $60,8 a$ & $63,7 \mathrm{~b}$ \\
\hline 7 & $06: 00 \mathrm{PM}$ & $38,50 \mathrm{~b}$ & $39,98 b$ & $32,80 a$ & $39,6 a$ & $54,8 \mathrm{a}$ & $53,7 a$ \\
\hline
\end{tabular}

a-b: (P 0,01); a-c: (P 0,05) y a-a: (P 0,05)

(Comparación Auxiliar)

\section{Indices fisiológicos}

\section{Temperatura rectal}

En principio, el comportamiento de la temperatura rectal durante el período diurno para vacas en lactación y terneros, fue diferente $(\mathrm{P} \leq 0.05)$, aunque en ambos grupos de animales se observó un incremento $(\mathrm{P} \leq 0,001)$ a través del período diurno más crítico en términos de tensión calórica. La temperatura rectal para vacas y terneros a las 06:00 am fue 37,78 y $38,04^{\circ} \mathrm{C}$; sin embargo, para las 10:00 am se había incrementado en 1,28 y $1,44^{\circ} \mathrm{C}$ respectivamente. La máxima temperatura se presentó a las $12: 00 \mathrm{md}\left(39,40^{\circ} \mathrm{C}\right)$ en las vacas y a las 02:00 pm $\left(39,73^{\circ} \mathrm{C}\right)$ en los terneros (Cuadro 3$)$. El índice de temperatura rectal a través del día presentó una tendencia cuadrática en las vacas en lactación $(\mathrm{P} \leq 0,01)$ y cúbica en los terneros $(\mathrm{P} \leq 0,1)$ como se indica en la Figura 1 . Se observó que el estado de hipertermia se mantuvo por más tiempo en los terneros que en las vacas; indicando que existe un diferencial en la necesidad de regulación térmica. Al respecto, las vacas en lactación tienen una mayor producción de calor debido al propio proceso de la biosíntesis láctea, por lo cual la temperatura corporal es regulada de manera más eficiente debido a la mayor necesidad de disipación calórica. A pesar de que la temperatura corporal es debidamente regulada por varios procesos complejos donde participan el hipotálamo, los sensores térmicos, la unidad termostática corporal, los mecanismos de producción y pérdida calórica (Bianca 1973; Anderson, 1984); pudo observarse que en vacas lecheras cruzadas en lactación y en terneros se produjo un incremento de la temperatura rectal durante el período diurno en la época seca. Esto indica que el nivel de tensión calórica de tipo climático es lo suficientemente fuerte como para alterar la temperatura corporal en animales lecheros cruzados en lactación y crecimiento, a
* Subperíodo diumo referencial para los patrones fisiologicos.

pesar de la influencia cebuina como parte de una alternativa de tolerancia al clima tropical.

\section{Frecuencia respiratoria}

El índice respiratorio presentó un comportamiento heterogéneo a través del dia para los animales en lactación y crecimiento ( $\mathrm{P} \leq 0,001)$.El mismo resultó ser el parámetro fisiológico más alterado. La magnitud normal tomada a las 06:00 am para vacas y terneros fue de 22 y 24 respiraciones/ min; sin embargo, el mismo a las 10:00 am fue de 60 y 68 respiraciones/min; y la máxima magnitud alcanzada fue de 76 y 81 (Cuadro 3). El comportamiento diurno del índice respiratorio en vacas en lactación fue cuadrático $(\mathrm{P} \leq 0,01)$ y en los terneros cúbico $(\mathrm{P} \leq 0,001)$. En general, las vacas mostraron superioridad en el comportamiento respiratorio (Figura 2), aún en las horas de mayor tensión calórica, aunque en ambos estados fisiológicos (lactación y crecimiento) ocurrió un incremento apreciable del índice respiratorio sobre el patrón fisiológico encontrado. Se observó polipnea: aumento de la frecuencia respiratoria al principio; no obstante, en las horas de la tarde los animales presentaron un estado transitorio de hipernea térmica al realizar respiraciones más profundas de lo mostrado en las primeras horas de sometimiento a la tensión calórica. Al combinar los cambios en el índice respiratorio y la temperatura rectal con los elementos climáticos se observó que las mayores alteraciones se produjeron en la zona crítica de tensión calórico-climática (Cuadro 2). Esta alteración apreciable de la frecuencia respiratoria sugiere que el ganado lechero cruzado en lactación y crecimiento es susceptible al efecto calóricotensivo de la época seca. Entre otras variables fisiológicas se tomó el índice cardíaco, el cual fue aumentado a través del dia en las vaca'i en lactación y terneros $(P \leq 0,001)$, pero no se presentaron diferencias entre ambos estados 
fisiológicos $(\mathrm{P} \leq 0,05)$. Se observó 53 y 52 respiraciones/min para vacas y terneros con un máximo en el día de 65 y 63 respectivamente. La mayor frecuencia cardíaca coincidió con los mayores índices de temperatura rectal, frecuencia respiratoria y tensión calórica respectivamente.

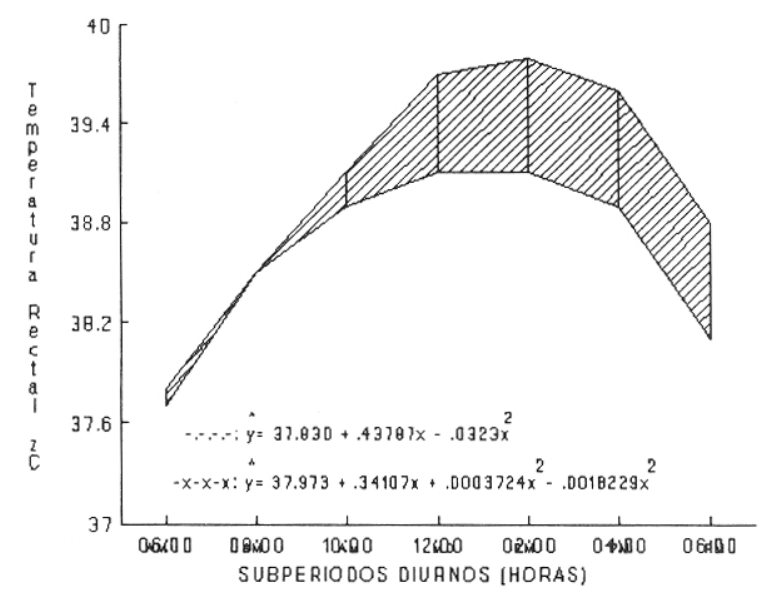

Figura 1. Tendencia regresiva de la temperatura retal en ganado lechero cruzado en lactación (-.-.-) y crecimiento (x.x.x) en el periodo diurno de la época seca.

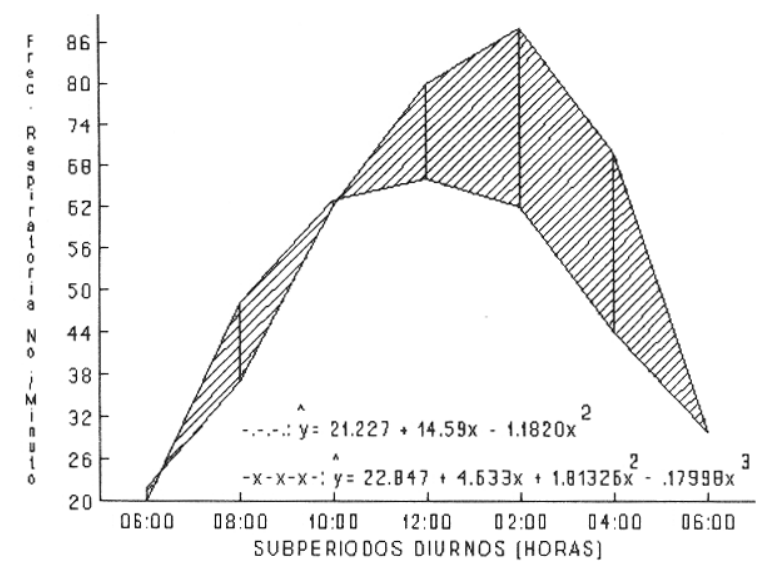

Figura 2. Tendencia de la frecuencia respiratoria en ganado lechero acebuado en la lactación (---) y crecimiento (x.x.x) sometido a tensión calórica en la época seca.

\section{Estado general de los animales}

En las horas de la mañana tanto las vacas como los terneros presentaron un estado y apariencia normal. Sin embargo, en las horas del período crítico en el día, particularmente entre 12:00 md y 04:00 pm se observó ligera salivación, jadeo, hipertermia, polipnea, hipernea térmica transitoria, agotamiento general, cansancio y cambios en la conducta general de los animales incluyendo las vacas en lactación y los terneros.

\section{CONCLUSIONES}

1. La caracterización microclimática en base a la radiación solar, temperatura ambiental e índice temperatura-humedad para bovinos indicó que el período diurno de la época seca es en efecto el período representativo de la tensión calórica efectiva de tipo climático, en especial entre 10:00 am y 04:00 pm.

2. Las vacas lecheras cruzadas en lactación y los terneros fueron afectados por la tensión calórica del período diurno, por lo cual la temperatura rectal yel índice respiratorio sufrieron incrementos sobre el patrón fisiológico, indicando tanto el nivel de tensión climático como la susceptibilidad de los animales respectivamente.

3. Al comprometerse la fisiología general del ganado lechero cruzado en lactación y crecimiento como parte de los efectos agudos, debe destacarse que también se comprometen aquellas funciones como la lactación, el crecimiento, la reproducción y gestación, lo cual merma el desempeño bioproductivo, por lo cual el manejo animal debe incluir el elemento microambiental.

\section{LITERATURA CITADA}

ANDERSON, B.E. 1984. Temperature regulation and environmental Physiology. In Dukes Physiology of Domestic Animals. Melvin J. Sweson ed. Comell Univ.Press. Ithaca. New York, USA. pp. 719-727.

BEEDE, D. K. et al. 1986. Potential Nutritional Strategies for intensively managed Cattle during thermal Stress. J. Animal Sci. 62: 543-554.

BlANCA, W. 1973. Termorregulación. In Adaptación de los Animales Domésticos. Hafez E.S, ed. Editorial Labor. pp.135-162.

BlANCA, et al., 1985. Efeccts of climatie and Management Factors on Conception Rate of Dairy Cattle in Subtropical Environment. J. Dairy Sci. 68: 78-85.

BULLOCK, J.; et al., 1984. Acid-Base Physiology. In: Physiology. John Willey \& Sons. New York, USA. pp. 223-263.

COLLIER, R. J., et al. 1982. Influences of Environment and its modifications of Dairy Animal Health and Production. 1. Dairy Sci. 65:2213-2227. 
COLLIER, R.J. et al. 1978. Patrones fisiológicos diarios en vacas bajo sombra. In: Duodécima Conferencia Anual sobre Ganadería y Avicultura en América Latina/IFAS. University of Florida, Gainesville Florida, U.SA. pp. E1E7.

CURTIS, S.E. 1981. Control and Integration of termorregulatory. Processes. In: Enviromental Management in Animal Agriculture. Animal environment Services. Illinois, USA. pp. 6-1 a 6-12.

FOLK, G. E. 1973. Medición de las Respuestas Fisiológicas a estímulos ambientales. In: Adaptación de los animales Domésticos, E.SE Hafez (ed). Editorial Labor. pp. 457476.

GILL, J.L. 1978. Split-Plot Designs. In: Design and Analysis of Experiments in the animal and Medical Sciences. Volume
2. The Iowa State Univesity Press/Ames, Iowa, USA. pp. 203-220.

HAFEZ, E. S. E. 1973. Efecto del medio ambiente sobre la producción de leche. In: Adaptación de los animales domésticos. Editorial Labor.

REECE, W. O. 1984. Respiration in mammals. In: Duckes Physiology of Domestic Animal by Melvin J. Swenson (ed). Cornell University Press. Ithaca, New York, U.S.A. pp. 226-254.

THOMPSON, G. E. 1985 . Respiratory System. In: Principies of Stress Physiology. Yousef, n k. (ed). pp. 155-161.

YOUSEF, M. K. 1985. Thermoneutral zone. In: Principies of Stress Physiology, Volume 1. Academic Press. USA. pp. 9-14, 67-69. 